\title{
Parasitosis intestinal y estado inmunológico en pacientes adultos con infección por VIH del Centro Médico Naval "Cirujano Mayor Santiago Távara”
}

\author{
Silvana Vergaray ${ }^{1}$; Rodrigo Corcuera-Ciudad ${ }^{2,3}$; Rosmery Paima-Olivari ${ }^{2,3}$; Fernando M. Runzer-Colmenares* ${ }^{2,4,5}$
}

RESUMEN

Objetivo: Describir los tipos parasitosis intestinales y el estado inmunológico en pacientes portadores del virus de la inmunodeficiencia humana $(\mathrm{VIH}+)$.

Materiales y métodos: Se realizó un estudio descriptivo, comparativo y retrospectivo, a través de la selección de 81 historias clínicas pertenecientes al Servicio de Infectología del Centro Médico Naval “Cirujano Mayor Santiago Távara”, con el permiso de las autoridades del centro. Las historias contaban con el único criterio de inclusión considerado en la investigación que fue diagnóstico de infección por VIH. Los datos que se obtuvieron fueron los resultados de examen coproparasitológico y citometría de flujo para linfocitos T CD4, la presencia o ausencia de sintomatología intestinal, y pertenencia al programa de tratamiento antirretroviral de alta actividad (TARGA) o al tratamiento antirretroviral convencional.

Resultados: El 37 \% presentaron parasitosis intestinal. Se observó con mayor frecuencia a Entamoeba coli (20 \%), que es no patógeno para el ser humano. Entre los patógenos hallados se encuentran Isospora belli (15,6 \%), Giardia lamblia (15,6 \%), Blastocystis hominis (11,2 \%) y Cryptosporidium sp. (11,2\%). El 32,1\% de los casos presentaron diarrea, mientras que el 67,9 \% fueron asintomáticos. En el grupo de asintomáticos, el promedio de CD4 es de 280 células/ $\mu \mathrm{L}$, mientras en los que tuvieron diarrea fue de 195 células/ $\mu \mathrm{L}$.

Conclusiones: Los enteroparásitos Isospora belli, Cyclospora cayetanensis, Blastocystis hominis son hallados con mayor frecuencia en recuentos de CD4 < 199 células/uL. Los parásitos no patógenos Entamoeba coli, Endolimax nana, Trichomonas hominis, Chilomastix mesnili se encuentran con mayor frecuencia en pacientes con recuentos de CD4 >200 células/uL.

Palabras clave: Infecciones por VIH; Parasitosis intestinales; Diarrea (Fuente: DeCS BIREME).

\section{Parasitic intestinal diseases and immune status in adult patients with HIV infection at the Peruvian Naval Medical Center "Cirujano Mayor Santiago Távara"}

ABSTRACT

Objective: To describe the types of parasitic intestinal diseases and the immune status in human immunodeficiency virus-positive $(\mathrm{HIV}+)$ patients.

Materials and methods: A descriptive, comparative and retrospective study was carried out through the selection of 81 medical records from the Infectious Disease Service of the Naval Medical Center "Cirujano Mayor Santiago Távara", with the authorization of the pertinent authorities. Said medical records included the only inclusion criterion considered in the research: the diagnosis of HIV infection. The following data was obtained: results from a copro-parasitological examination and a flow cytometry for CD4 T lymphocyte count, presence or absence of gastrointestinal symptoms, and participation in the Highly Active Antiretroviral Therapy (HAART) program or the conventional antiretroviral treatment program.

Results: Thirty-seven percent (37\%) of the patients presented parasitic intestinal diseases, with Entamoeba coli being the most frequently observed parasite $(20 \%)$, which is not pathogenic for humans. Among the pathogens, Isospora belli (15.6\%), Giardia lamblia (15.6\%), Blastocystis hominis (11.2\%) and Cryptosporidium sp. (11.2\%) were found. Thirty-two point one percent $(32.1 \%)$ of the subjects had diarrhea, while $67.9 \%$ were asymptomatic. In the asymptomatic group, the average CD4 cell count was 280 cells/ $\mu \mathrm{L}$, while in those with diarrhea it was 195 cells/ $\mu \mathrm{L}$.

Conclusions: Enteroparasites Isospora belli, Cyclospora cayetanensis and Blastocystis hominis are most frequently found at CD4 cell counts $<199$ cells/uL. Non-pathogenic parasites Entamoeba coli, Endolimax nana, Trichomonas hominis and Chilomastix mesnili are most frequently found in patients with CD4 cell counts $>200$ cells $/ \mu \mathrm{L}$.

Keywords: HIV infections; Parasitic intestinal diseases; Diarrhea (Source: MeSH NLM).

1. Hospital Santa Rosa. Lima, Perú.

2. Universidad Científica del Sur, Facultad de Ciencias de la Salud. Lima, Perú.

3. Sociedad Científica de Estudiantes de Medicina. Lima, Perú.

4. Universidad de San Martín de Porres, Facultad de Medicina Humana. Lima, Perú.

5. Centro Médico Naval "Cirujano Mayor Santiago Távara”. Callao, Perú.

* Autor corresponsal. 


\section{INTRODUCCIÓN}

Uno de los principales problemas que afronta la humanidad es la pandemia producida por la infección del virus de la inmunodeficiencia humana $(\mathrm{VIH})$. Este virus produce disminución de la respuesta inmune del paciente debido a que posee afinidad por las células que presentan receptor CD4 en su membrana, como los linfocitos T, macrófagos-monocitos y células dendríticas; lo que ocasiona que el organismo sea vulnerable a las infecciones propias de un estado inmunosuprimido ${ }^{(1)}$.

El recuento de linfocitos T CD4 refleja la capacidad inmunológica de la persona infectada, así como también predice con mayor exactitud la progresión de la infección. En muchos trabajos de investigación se ha tomado como parámetro de CD4 el recuento menor de 200 células/ $\mu \mathrm{L}$ para la presencia de infecciones oportunistas, en este caso, por parásitos intestinales ${ }^{(2)}$.

En un paciente con infección VIH con sintomatología gastrointestinal, como diarrea, dolor abdominal, fiebre, hemorragia digestiva, odinofagia, disfagia, náusea, vómitos y, en casos graves, pérdida de peso, es muy importante establecer el diagnóstico etiológico a través de la realización de coprocultivos y exámenes coproparasitológicos en búsqueda de bacterias y enteroparásitos ${ }^{(3)}$. En caso los resultados de estos exámenes sean negativos, se debe proceder a la obtención de una biopsia rectal ante la posibilidad de etiología secundaria a citomegalovirus, micobacterias atípicas o Microsporidium. Ante sintomatología persistente por más de un mes, es probable que el agente causal sea el propio $\mathrm{VIH}{ }^{(4)}$, mediante cambios producidos en la mucosa gastrointestinal, como atrofia de las vellosidades intestinales ${ }^{(5)}$.

Los pacientes inmunocomprometidos son más susceptibles a parasitosis oportunistas. Se conoce que, a mayor disminución de la respuesta inmune, mayor prevalencia de parasitosis oportunistas ${ }^{(6)}$, que pueden causar una diarrea prolongada de carácter inespecífico, la que, si no recibe tratamiento de soporte adecuado, podría ocasionar la muerte del paciente.

En el Perú no se han encontrado reportes de estudios entre parasitosis intestinal y el estado inmune en los pacientes $\mathrm{VIH}(+)$ en terapia antirretroviral, por lo que se considera de especial relevancia establecer la relación entre ambos, así como observar si la prevalencia de parasitosis en pacientes $\mathrm{VIH}(+)$ es similar a la encontrada en otros países, por lo que el objetivo de nuestro estudio fue describir la frecuencia de parasitosis en pacientes $\mathrm{VIH}(+)$.

\section{MATERIALES Y MÉTODOS}

\section{Diseño y población}

Estudio transversal, comparativo y retrospectivo. Se solicitó permiso para el acceso a las historias clínicas del Servicio de Infectología del Centro Médico Naval "Cirujano Mayor Santiago Távara" durante el 2006. Se seleccionaron 81 historias clínicas con el único criterio de inclusión del estudio, que fue diagnóstico de infección por VIH, se excluyeron a los pacientes con tratamiento profiláctico y con inmunosupresión de otra causa distinta a infección por $\mathrm{VIH}$, además de los menores de 18 años. El muestreo fue no probabilístico. De las historias reclutadas, se evidenciaron casos de participantes que presentaron nuevos episodios de diarrea o se les realizó nuevos exámenes coproparasitológicos cuyos resultados fueron positivos. Ante ello, fueron incluidos como casos que si cumplían con los criterios de inclusión/exclusión.

\section{Variables y mediciones}

Tipo de parasitosis, definido como la presencia, en la historia clínica, de un informe de laboratorio con el hallazgo de parasitosis intestinales; episodios diarreicos descritos en la historia clínica; conteo de células CD4 en los chequeos ambulatorios de consulta externa de Infectología y edad en años.

\section{Análisis estadístico}

El tamaño muestral de 102 participantes se calculó a partir del total del total de historias (150), se asumió una frecuencia de $28,88 \%$ de parasitosis en pacientes con VIH-SIDA según Barbosa et al., un efecto de diseño de 1 y un error del $5 \%$. Se empleó el software OpenEpi (Open Source Epidemiologic Statistics for Public Health). Los datos obtenidos de las historias clínicas fueron almacenados en el programa Microsoft Excel, y el análisis de los datos seleccionados se realizó con el programa SPSS 13.0. No se consideraron nombres ni códigos personales para cuidar la confidencialidad de la información. Los datos se analizaron mediante ANOVA Post Hoc Test, Chi-Cuadrado, frecuencia, Levenes's Test y T de Student.

\section{Consideraciones éticas}

El presente estudio fue realizado como parte de los requisitos de la obtención del título de médico cirujano de la autora principal y fue aprobado por el Comité de Ética de la Universidad Científica del Sur, así como por el Centro Médico Naval; no se utilizaron datos personales que puedan servir para identificar a los participantes del estudio. 


\section{RESULTADOS}

La edad mínima de los pacientes incluidos en el estudio fue 21 años y la máxima, 70, con un promedio de 39 años. El sexo masculino fue el predominante con un total de 72 casos $(88,9 \%)$ frente al sexo femenino con 9 casos $(11,1 \%)$.

Del total de la población estudiada, el 37 \% presentó parasitosis intestinal. Se encontró con mayor frecuencia Entamoeba coli (20\%), que se considera no patógeno para el ser humano. También se observan a otros no patógenos como Endolimax nana (4,4 \%), Trichomonas hominis (4,4\%), Chilomastix mesnili (2,2\%). Entre los patógenos hallados se encuentran Isospora belli $(15,6 \%)$, Cyclospora cayetanensis (8,9\%), Giardia lamblia (15,6 $\%)$, Blastocystis hominis (11,2\%) y Cryptosporidium spp. (11,2\%). El 2,7\% de la población presentó más de una parasitosis en el mismo episodio diarreico. Además, el 17,2\% presentó más de un episodio de diarrea y/o presencia de parásitos intestinales.

Se agrupó a los pacientes según el recuento de CD4 en $<199$ células/ $\mu \mathrm{L}$ y $>200$ células/ $\mu \mathrm{L}$, y se observó mayor frecuencia de parasitosis intestinal en el primer grupo (Figura 1).

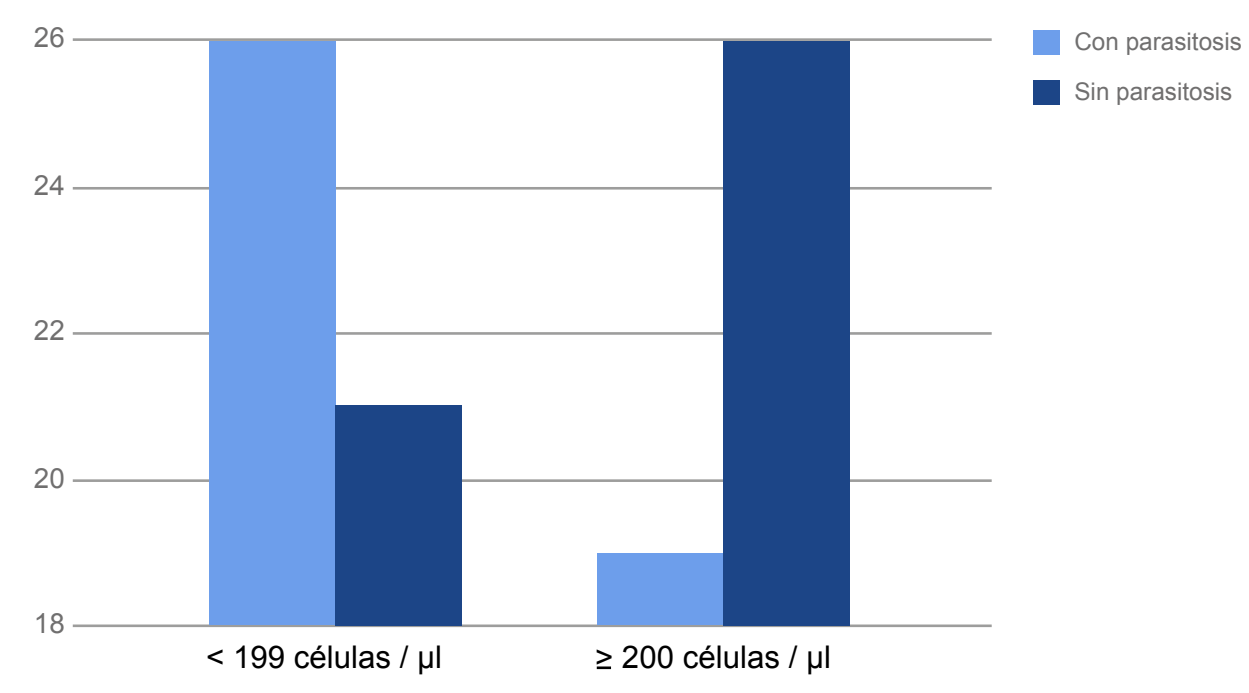

Figura 1. Relación entre parasitosis y recuento de CD4

El $32,1 \%$ de los casos presentaron diarrea, mientras que el 67, $9 \%$ fue asintomático. En el grupo de asintomáticos, el promedio de CD4 fue de 280 células/ $\mu \mathrm{L}$, mientras en los que tuvieron diarrea fue de 195 células/ $\mu \mathrm{L}$. Se encontró una diferencia estadísticamente significativa para esta asociación. La relación entre parasitosis intestinal y sintomatología intestinal también fue estadísticamente significativa, de 39 casos con diarrea, 27 presentaron parasitosis.

\section{DISCUSIÓN}

El promedio de edad de los pacientes fue de 39 años (mínima, 21 y máxima, 70), lo cual demuestra que la infección se presenta en distintos grupos etarios. Los menores de 18 años no fueron incluidos en el estudio. Respecto a la frecuencia según el sexo, se encuentra predominancia en el sexo masculino $(88,9 \%)$ frente al sexo femenino $(11,1 \%)$.

Respecto a los parásitos hallados en este estudio, se encontraron resultados similares en América Latina, sin embargo, a diferencia de este estudio, no siempre se incluyeron a pacientes que presentaron diarrea ${ }^{(5,6)}$. En otras investigaciones, los resultados hallados concordaron con los nuestros, pero cabe destacar que trabajaron con pacientes con diarrea ${ }^{(7)}$. 
Dentro de la población se han encontrado dos casos en los que se ha aislado más de una especie de parásito en la misma muestra de heces. En estos dos casos los pacientes han presentado diarrea y además recibían TARGA. Los parásitos hallados fueron Isospora belli con Entamoeba coli y Dipylidium caninum con Toxocara canis, ambos casos con recuento de CD4 menor de 200 células $/ \mu \mathrm{L}$.

Entre los pacientes seleccionados para el trabajo no se hallaron casos de infecciones gastrointestinales por enteroparásitos, a pesar de haber tenido pacientes con enfermedad diarreica por parásitos patógenos e inmunosupresión avanzada ${ }^{(8)}$.

En el estudio sobre recuento de CD4, a los pacientes se les divide en dos grupos: $C D 4<199$ células/ $\mu \mathrm{L}$ y CD4 > 200 células $/ \mu \mathrm{L}$. Se ha podido apreciar mayor frecuencia de parasitosis oportunistas en el primer grupo. Se hallaron Cryptosporidium parvum, Cyclospora cayetanensis, Blastocystis hominis, el más frecuente fue Isospora belli, lo que no concuerda con varios estudios en donde la parasitosis más frecuente es Cryptosporidium spp. ${ }^{(1,9)}$. En el segundo grupo, el parásito intestinal predominante fue Entamoeba coli; además, aparecen otros parásitos como Giardia lamblia y Endolimax nana. La presencia de esta última es similar en una persona inmunocompetente $y$, en muchas ocasiones, no llega a producir enfermedad, como es el caso de Giardia lamblia. La diferencia hallada en los parásitos intestinales entre ambos grupos de CD4, puede explicarse porque estos microorganismos desencadenan reacciones distintas en el hospedero, que logra neutraliza a los patógenos pero que no reacciona de la misma forma con los parásitos no patógenos.

Respecto a la presencia de sintomatología intestinal, tomamos como referencia a la diarrea por ser la manifestación más frecuente en estos pacientes y el síntoma principal de las parasitosis intestinales. Del total de casos, se evidencia que más de la mitad tiene un examen coproparasitológico positivo $(69,2 \%)$, aunque hay un porcentaje de $28,6 \%$ que presenta parasitosis asintomática. Esto puede explicarse porque el paciente presenta un recuento de CD4 no muy alterado o la parasitosis no es patógena; sin embargo, no podemos dejar de considerar al factor tratamiento antirretroviral que puede influir en la aparición de diarrea (efecto de los medicamentos), y la aparición de infecciones oportunistas ${ }^{(10)}$.

Se ha podido evidenciar que la diarrea es más frecuente en el grupo de pacientes con recuento de $C D 4<199$ células/ $\mu \mathrm{L}$, de acuerdo con trabajos en los que se analiza la estrecha relación entre la baja de CD4 y la presencia de diarrea en pacientes $\mathrm{VIH} \mathrm{(+)}{ }^{(6)}$. Se debe tomar en cuenta en cuenta que no solo las parasitosis pueden producir diarrea; se debe incluir a las infecciones por virus, bacterias y hongos, además de las producidas por intoxicaciones y medicamentos.

A medida que se ha incluido el tratamiento antirretroviral, ya sea constituido por una sola droga o al pertenecer al programa TARGA, se ha podido encontrar diferencia estadísticamente significativa en relación al recuento de CD4 entre los casos con y sin tratamiento, el promedio de recuento de CD4 del grupo de tratamiento antirretroviral, ya sea con TARGA o con monodroga, es inferior al promedio encontrado en el grupo de sin tratamiento antirretroviral. Esto se debe a que uno de los indicadores para iniciar TARGA es el recuento de CD4 por debajo de 200 células $/ \mu \mathrm{L}^{(10)}$.

Se considera conveniente hacer el seguimiento de los pacientes del programa TARGA, para poder observar la evolución de la infección con la influencia del tratamiento antirretroviral.

En conclusión, el recuento de linfocitos TCD4 <199 células $/ \mathrm{mm} 3$ predisponen a mayor susceptibilidad de contraer infecciones por enteroparásitos patógenos, lo que confirma la hipótesis inferencial. Los enteroparásitos Isospora belli, Cyclospora cayetanensis, Blastocystis hominis son hallados con mayor frecuencia en recuentos de CD4 <199 células/uL. Por otro lado, los parásitos no patógenos Entamoeba coli, Endolimax nana, Trichomonas hominis, Chilomax mesnili se encuentran con mayor frecuencia en pacientes con recuentos de CD4 >200 célula/uL. Además, se encontró que recuentos de CD4<199 células/uL muestran una fuerte asociación con diarrea.

\section{REFERENCIAS BIBLIOGRÁFICAS}

1. Clarridge KE, Blazkova J, Einkauf K, Petrone M, Refsland EW, Justement JS, et al. Effect of analytical treatment interruption and reinitiation of antiretroviral therapy on HIV reservoirs and immunologic parameters in infected individuals. PLoS Pathog. 2018; 14(1): e1006792.

2. Lee CY, Tseng YT, Lin WR, Chen YH, Tsai JJ, Wang, et al. AIDS-related opportunistic illnesses and early initiation of HIV care remain critical in the contemporary HAART era: a retrospective cohort study in Taiwan. BMC Infect Dis. 2018; 18(1): 352.

3. Amoo JK, Akindele AA, Amoo AO, Efunshile AM, Ojurongbe TA, Fayemiwo SA, et al. Prevalence of enteric parasitic infections among people living with HIV in Abeokuta, 
Nigeria. Pan Afr Med J. 2018; 30(66).

4. Goldsby RA. Inmunología. 5a ed. México: McGraw-Hill Interamericana; 2004.

5. Fernández $\mathrm{Na}$, Combo A, Zanetta E, Acuña A, Gezuele E. Primer diagnóstico de microsporidiosis humana en Uruguay. Rev Méd Urug. 2002; 18(3): 251-55.

6. Martínez I, Ayllón L, Benítez X. Cyclospora cayetanensis: Presentación de 20 casos. Rev Cubana Pediatr. 2002; 74(2): 178-81.

7. Brink A-K, Mahé $C$, Watera $C$, Lugada E, Gilks $C$, Whitworth $J$, et al. Diarrhoea, CD4 Counts and Enteric Infections in a Community-Based Cohort of HIV-Infected Adults in Uganda. J Infect. 2002; 45(2):99-106.

8. Capó V, Barrero M, Velázquez B, Luzardo C, Martínez A, Alujas Z. Diagnóstico de coccidias y microsporas en muestras de heces diarreicas de pacientes cubanos seropositivos al VIH: Primer reporte de microsporas en Cuba. Rev Cubana Med Trop. 2003; 55(1): 14-18.

9. Cimerman S, Cimerman B, Lewi D. Enteric parasites and AIDS. Sao Paulo Med J. 1999; 117(6): 266-73.

10. Barcelos NB, Silva LFE, Dias RFG, Menezes HR, Rodrigues RM. Opportunistic and non-opportunistic intestinal parasites in HIV/AIDS patients in relation to their clinical and epidemiological status in a specialized medical service in Goiás, Brazil. Rev Inst Med Trop Sao Paulo. 2018; 60:e13.
Fuentes de financiamiento:

Este artículo ha sido financiado por los autores

Conflictos de interés:

El quinto autor con iniciales FMRC, labora en el Centro Médico Naval "Cirujano Mayor Santiago Távara". Callao, Perú; al momento de realizar dicho artículo.

\section{Correspondencia:}

Fernando M. Runzer Colmenares

Dirección: Caminos del Inca 556, Surco. Lima, Perú.

Teléfono: +51942190471

Correo electrónico: frunzer@cientifica.edu.pe

\section{Recibido: 16 de setiembre de 2018 .}

Evaluado: 23 de setiembre de 2018 .

Aprobado: 24 de octubre de 2018

๑ La revista. Publicado por Universidad de San Martín de Porres, Perú. (c) Br Licencia de Creative Commons Artículo en acceso abierto bajo términos de Licencia Creative Commons Atribución 4.0 Internacional. (http://creativecommons.org/licenses/by/4.0/)

\section{ORCID iDs}

Silvana Vergaray Rodrigo Corcuera Ciudad

Rosmery Paima Olivari

Fernando M. Runzer Colmenares

(iD) https://orcid.org/0000-0001-8409-2093

(iD) https://orcid.org/0000-0002-3936-0295

(iD) https://orcid.org/0000-0002-5217-5967

(i) https://orcid.org/0000-0003-4045-0260 